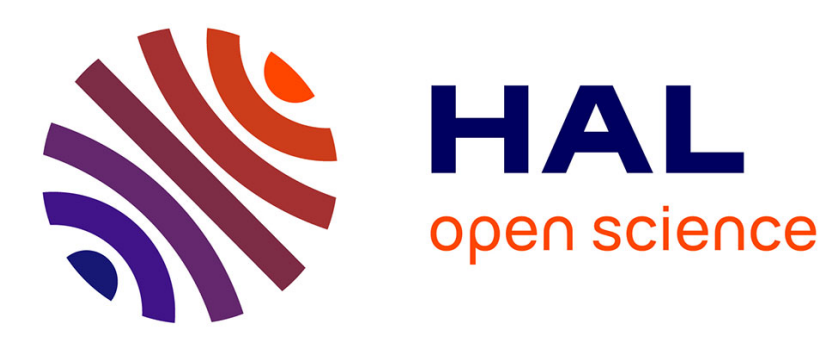

\title{
Strain simulation of steel during a heating-cooling cycle including solid-solid phase change
}

Stéphane Valance, Michel Coret, Alain Combescure

\section{To cite this version:}

Stéphane Valance, Michel Coret, Alain Combescure. Strain simulation of steel during a heatingcooling cycle including solid-solid phase change. European Journal of Mechanics - A/Solids, 2007, 26

(3), pp.460-473. 10.1016/j.euromechsol.2006.11.001 . hal-01007324

\section{HAL Id: hal-01007324 \\ https://hal.science/hal-01007324}

Submitted on 3 Jun 2017

HAL is a multi-disciplinary open access archive for the deposit and dissemination of scientific research documents, whether they are published or not. The documents may come from teaching and research institutions in France or abroad, or from public or private research centers.
L'archive ouverte pluridisciplinaire HAL, est destinée au dépôt et à la diffusion de documents scientifiques de niveau recherche, publiés ou non, émanant des établissements d'enseignement et de recherche français ou étrangers, des laboratoires publics ou privés. 


\title{
Strain simulation of steel during a heating-cooling cycle including solid-solid phase change
}

\author{
S. Valance, M. Coret, A. Combescure \\ INSA/LaMCoS, UMR/CNRS 5514, Bât. J. d'Alembert, 20, av A. Einstein, 69621 Villeurbanne Cedex, France
}

\begin{abstract}
Designed for the simulation of transformation induced plasticity in steels occurring on cooling, the model first proposed by Leblond in the middle of 80's works well for constant uni-axial stress test. But further experimental investigation have shown that for non-proportional multi-axial test, the quality of the model is worse. The purpose of this paper is to improve this model through the removal of the hypothesis that plastic flow for the Greenwood-Johnson mechanism occurs only in the 'weaker' phase. The improvement, leading to better agreement, enables to use the model on cooling, and shows that for TRIP plastic flowing in harder phase is almost important, even if it is lower than in the 'weak' phase.
\end{abstract}

Keywords: Transformation induced plasticity (TRIP); Solid phase transformation; Greenwood and Johnson effect; Leblond's model

\section{Introduction}

The model presented below is applied to the simulation of transformation induced plasticity (TRIP) for low carbon steels. Material behavior taken into account are elastic with either ideal plasticity or linear kinematic hardening. The focus of this model is to relate stress, temperature and phase proportion to strain during the transformation.

For steels undergoing a solid-solid phase transformation, TRIP appears. This phenomenon is a plastic flow occurring when an external load is applied during the transformation. This occurs even if this load is small regarding yield stress of the 'weaker' phase. Greenwood and Johnson (1965) and Magee (1970) give two complementary explanations to this phenomenon:

- orientation of the local plastic flow due to phase volume incompatibility by external loading (Greenwood and Johnson mechanism);

- preferred orientation of the martensite plates arising from the external loading (Magee mechanism).

From a metallurgical point of view, Greenwood and Johnson mechanism is related to diffusional transformation and Magee effect is related to displacive transformation occurring for martensitic transformation involving Bain strain. 
Of course these mechanisms are not exclusive, but experimental works show preponderant relation between them (Bhadeshia, 1995).

Modeling of Greenwood and Johnson transformation plasticity phenomenon has mainly been developed by Leblond et al. (1986a, 1986b, 1989b, 1989a) and by Fischer (1990). According to recent experimental work of Coret et al. (2003) and to analytical work of Taleb and Sidoroff (2002), Leblond model give accurate results. However this model is not designed to describe the behavior of the 'harder' phase. Therefore, the goal of the following work is to up grade the so called Leblond model by taking into account the possibility of plastic flowing in the classical named 'harder' phase. Indeed, in agreement with Greenwood and Johnson (1965) work on TRIP, Leblond et al. (1986b) has removed the possibility of plastic flow of the 'harder' phase.

Since model used is similar to Leblond's one, we will first present how to relate macroscopic (structure) scale to microscopic scale (grain scale) in the sense of Leblond. Then we will present the micromodel which enable to take plasticity into account in the harder phase. Application to ideal elasto-plastic case and how to mix it up with the precedent approach is done. Then, an extension to the linear kinematic hardening case is also introduced and at last, a comparison with experiments is done.

\section{A summary of the Leblond model}

Since this paper is strongly linked to Leblond model, this part presents the main feature of the model (Leblond et al. 1986a, 1986b, 1989b, 1989a). We will take for instance a transformation occurring on cooling from a weak phase $\gamma$ to a hard phase $\alpha$, the notation $\lambda$ will be reserved for denoting either the $\alpha$ or $\gamma$ phase. In the whole paper $z$ will denote the $\alpha$ phase proportion. In his model, Leblond et al. (1986a) uses a macroscopic scale, capital notations in this paper, and a microscopic scale, lowercase notations. The macroscopic one enables to separate the contribution of different phenomenon that are:

- classical plasticity related to mechanical load variation;

- classical plasticity related to thermal load variation;

- transformation plasticity related to phase proportion variation implying Greenwood and Johnson and Magee effects.

Let us now introduce hypothesis:

Hypothesis 1. The microscopic elastic compliance tensor $\mathbf{m}$ may be equated to the macroscopic overall elastic compliance tensor $\mathbf{M}$.

Under Hypothesis 1, one obtains macroscopic plastic strain rate $\dot{E}^{\text {p }}$ decomposition given by Eq. (2.1), into contribution of classical plasticity strain rate due to external load $\Sigma$ (stress tensor) variation, $\dot{E}_{\Sigma}^{\mathrm{cp}}$, classical plasticity strain rate due to temperature variation $\dot{T}, \dot{E}_{T}^{\mathrm{cp}}$, and transformation plasticity due to phase variation, $\dot{E}^{\text {tp }}$. Next, from a thermodynamical approach, we could get homogenization relations between macroscopic strain rate and microscopic plastic strain $\epsilon^{\mathrm{p}}$ variation, given by Eq. (2.1).

$$
\dot{E}^{\mathrm{p}}=\dot{E}_{\Sigma}^{\mathrm{cp}}+\dot{E}_{T}^{\mathrm{cp}}+\dot{E}^{\mathrm{tp}}
$$

with:

$$
\left\{\begin{array}{l}
\dot{E}_{\Sigma}^{\mathrm{cp}}=\left[(1-z)\left\langle\frac{\delta \epsilon^{\mathrm{p}}}{\delta \Sigma}\right\rangle_{V_{\gamma}}+z\left\langle\frac{\delta \epsilon^{\mathrm{p}}}{\delta \Sigma}\right\rangle_{V_{\alpha}}\right] \dot{\Sigma}, \\
\dot{E}_{T}^{\mathrm{cp}}=\left[(1-z)\left\langle\frac{\delta \epsilon^{\mathrm{p}}}{\delta T}\right\rangle_{V_{\gamma}}+z\left\langle\frac{\delta \epsilon^{\mathrm{p}}}{\delta T}\right\rangle_{V_{\alpha}}\right] \dot{T}, \\
\dot{E}^{\mathrm{tp}}=\left[(1-z)\left\langle\frac{\delta \epsilon^{\mathrm{p}}}{\delta z}\right\rangle_{V_{\gamma}}+z\left\langle\frac{\delta \epsilon^{\mathrm{p}}}{\delta z}\right\rangle_{V_{\alpha}}+\left\langle\Delta \epsilon_{\alpha \gamma}^{\mathrm{p}}\right\rangle_{F\left(U_{n}\right)}\right] \dot{z} .
\end{array}\right.
$$

Remark 1. In the preceding equations, the 'derivative like' $\delta \cdot / \delta \cdot$ notation was used to emphasize that $\dot{\epsilon}$ is not only function of $\Sigma, T, z$. 
The last term of $\dot{E}^{\text {tp }}$, which is an average on the front, is linked to Magee mechanism and the two first, average on $\alpha, V_{\alpha}$, and $\gamma, V_{\gamma}$, phase volume, explain the Greenwood and Johnson effect. Since the model is only interesting in diffusional transformation, the Magee mechanism will be neglected for the model. Next step is the removing of 'weak' phase terms under Hypothesis 2:

Hypothesis 2. For small or moderately high applied stresses, the $\gamma$ phase is entirely plastic, but the $\alpha$ phase remains elastic, or its plastic strain rate remains always much smaller than the $\gamma$ phase's one.

This hypothesis first appears in Greenwood and Johnson (1965). It is justified by the fact that yield stress of $\gamma$ phase is much smaller than that of $\alpha$ phase. But, as will be discussed in Section 3, this justification could not be sufficient to remove this term. Finally, these hypothesis lead to Eqs. (2.3):

$$
\left\{\begin{array}{l}
\dot{E}^{\mathrm{tp}}=(1-z)\left\langle\frac{\delta \epsilon^{\mathrm{p}}}{\delta z}\right\rangle_{V_{\gamma}} \dot{z}, \\
\dot{E}_{T}^{\mathrm{cp}}=(1-z)\left\langle\frac{\delta \epsilon^{\mathrm{p}}}{\delta T}\right\rangle_{V_{\gamma}} \dot{T}, \\
\dot{E}_{\Sigma}^{\mathrm{cp}}=(1-z)\left\langle\frac{\delta \epsilon^{\mathrm{p}}}{\delta \Sigma}\right\rangle_{V_{\gamma}} \dot{\Sigma} .
\end{array}\right.
$$

From this point, Leblond begins a homogenization step to replace the preceding set of equations by a new one which only depends on the microscopic equivalent strain in order to get useful equations for the micro-mechanic model.

Hypothesis 3. Both phases obey the von Mises criterion and the Prandtl-Reuss flow rule.

Hypothesis 4. Interactions between $\delta \epsilon_{\gamma}^{\mathrm{eq}} / \delta \Sigma_{i j}$ and $s_{\gamma}$ which is the microscopic stress deviator in $\gamma$ phase, can be neglected.

Hypothesis 5. For small applied stresses, the macroscopic stress deviators $S_{\gamma}$ and $S_{\alpha}$ in phase $\gamma$ and $\alpha$ are almost equal and identical to the total macroscopic stress deviator $S$, i.e., the average value of the microscopic deviator over the whole representative volume $V$ :

$$
S_{\gamma}=S_{\alpha}=(1-z) S_{\gamma}+z S_{\alpha}=S .
$$

Hypothesis 6. $\Sigma^{\mathrm{eq}}=\left(\frac{3}{2} S_{i j} S_{i j}\right)^{1 / 2}$ denoting the macroscopic von Mises equivalent stress, $\dot{E}_{\Sigma}^{\mathrm{cp}}$ is non-zero only if $\Sigma^{\mathrm{eq}}$ varies, i.e.:

$$
\begin{aligned}
& \left\langle\frac{\delta \epsilon_{\gamma}^{\mathrm{eq}}}{\delta \Sigma_{i j}}\right\rangle_{V_{\gamma}} \dot{\Sigma}_{i j} \equiv\left\langle\frac{\delta \epsilon_{\gamma}^{\mathrm{eq}}}{\delta \Sigma_{\mathrm{eq}}}\right\rangle_{V_{\gamma}} \dot{\Sigma}_{\mathrm{eq}}, \\
& \left\{\begin{array}{l}
\dot{E}^{\mathrm{tp}}=\frac{3(1-z)}{2 \sigma_{\gamma}^{y}}\left\langle\frac{\delta \epsilon_{\gamma}^{\mathrm{eq}}}{\delta z}\right\rangle_{V_{\gamma}} S \dot{z}, \\
\dot{E}_{T}^{\mathrm{cp}}=\frac{3(1-z)}{2 \sigma_{\gamma}^{y}}\left\langle\frac{\delta \epsilon_{\gamma}^{\mathrm{eq}}}{\delta T}\right\rangle_{V_{\gamma}} S \dot{T}, \\
\dot{E}_{\Sigma}^{\mathrm{cp}}=\frac{3(1-z)}{2 \sigma_{\gamma}^{y}}\left\langle\frac{\delta \epsilon_{\gamma}^{\mathrm{eq}}}{\delta \Sigma^{\mathrm{eq}}}\right\rangle_{V_{\gamma}} S \dot{\Sigma}^{\mathrm{eq}} .
\end{array}\right.
\end{aligned}
$$

The last set of equation enables to relate macroscopic strain rate and microscopic strain variation. It remains to evaluate microscopic strain variation. It could be done by submitting a representative local zone to a variation of $z, T$, and $\Sigma$.

Since $z$ and $T$ are scalar quantities, the micro-model used to estimate $\dot{E}^{\text {tp }}$ and $\dot{E}_{T}^{\text {cp }}$ is based on a spherical geometry (Leblond et al., 1989b). On the other hand, since $\Sigma^{\text {eq }}$ is related to a tensorial quantity, a shearing micro-model and a traction micro-model are introduced (Leblond et al., 1986b). 

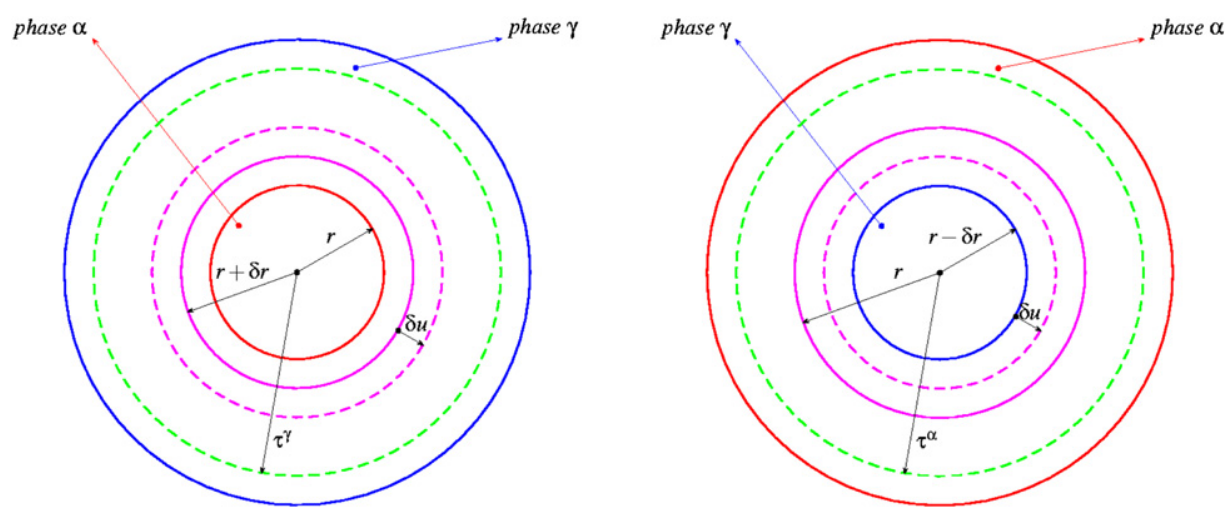

Fig. 1. Configuration $\gamma$ and $\alpha$ : schema of micro-models for the estimation of plastic flow in $\gamma$ and $\alpha$ phases.

Micro-model for the estimation of $\left\langle\frac{\delta \epsilon_{\gamma}^{\mathrm{eq}}}{\delta z}\right\rangle_{V_{\gamma}}$ and $\left\langle\frac{\delta \epsilon^{\mathrm{eq}}}{\delta T}\right\rangle_{V_{\gamma}}$. Micro-model (cf. Fig. 1) to estimate $\left\langle\frac{\delta \epsilon_{\gamma}^{\mathrm{eq}}}{\delta z}\right\rangle_{V_{\gamma}}$ and $\left\langle\frac{\delta \epsilon^{\mathrm{eq}}}{\delta T}\right\rangle_{V_{\gamma}}$ is made of two inclusive spheres of each materials which radius is evolving proportionally to phase proportion.

Mechanical problem to solve on these micro-model is defined through hypothesis and boundary conditions:

Hypothesis 7. In spherical micro-models, hypothesis used are listed below:

- external stress is neglected in comparison to the stress induced by transformation induced plasticity;

- both phases are expected to be ideal elasto-plastic materials.

Boundary conditions 1. Boundary conditions used to obtain $\epsilon^{\mathrm{eq}}$ in the micro model are:

- behavior at the center is smooth. Hence, no plastic flow occurs in the $\alpha$ phase without significant external load;

- continuity of the radial displacement on the boundary $\alpha-\gamma^{\mathrm{p}}$ and $\gamma^{\mathrm{p}}-\gamma^{\mathrm{el}}$;

- continuity of the radial stress on the boundary $\alpha-\gamma^{\mathrm{p}}$ and $\gamma^{\mathrm{p}}-\gamma^{\mathrm{el}}$;

- continuity of the circumferential stress on boundary $\alpha-\gamma^{\mathrm{p}}$;

- null radial stress on the external boundary of the $\gamma$ phase.

Solving the mechanical problem using equilibrium, the variation of local equivalent strain $\delta \epsilon^{\mathrm{eq}}$ in the plastified zone writes:

$$
\delta \epsilon^{\mathrm{eq}}(x)=\frac{6 \Delta \epsilon_{\gamma \alpha}^{\mathrm{th}} r^{2} \delta r}{x^{3}}
$$

where $x$ denote the radial coordinate and, $\Delta \epsilon_{\gamma \alpha}^{\mathrm{th}}=\epsilon_{\alpha}^{\mathrm{th}}-\epsilon_{\gamma}^{\mathrm{th}}$ is the difference of thermal strain between the two phases.

As the micro-model is assumed to be representative of a local zone, it allows access to homogenized quantities through integration. Taleb's work (Taleb and Sidoroff, 2002) on the plastification radius for the TRIP term, enables to reduce the integration on the only plastified zone, so that one gets Eqs. (2.6) and (2.7) for TRIP strain rates.

$$
\begin{aligned}
& \left\{\begin{array}{l}
\left\langle\frac{\delta \epsilon^{\mathrm{eq}}}{\delta z}\right\rangle_{V_{\gamma}}=-2 \frac{\Delta \epsilon_{\gamma \alpha}^{\mathrm{th}}}{1-z} \ln \left(z_{\gamma}\right) \quad \text { if } z \leqslant z_{\gamma} \\
\left\langle\frac{\delta \epsilon^{\mathrm{eq}}}{\delta z}\right\rangle_{V_{\gamma}}=-2 \frac{\Delta \epsilon_{\gamma \alpha}^{\mathrm{th}}}{1-z} \ln (z) \quad \text { if } z>z_{\gamma}
\end{array} \quad \text { with } z_{\gamma}=\frac{\sigma_{\gamma}^{y}}{\Delta \epsilon_{\gamma \alpha}^{\mathrm{th}}} \frac{4 \mu_{\gamma}+3 K_{\gamma}}{18 K_{\gamma} \mu_{\gamma}}\right. \\
& \left\langle\frac{\delta \epsilon^{\mathrm{eq}}}{\delta T}\right\rangle_{V_{\gamma}}=2 \frac{z}{1-z}\left(\alpha_{\gamma}-\alpha_{\alpha}\right) \ln (z)
\end{aligned}
$$

with $\alpha_{\lambda}$ the expansion coefficient of the $\lambda$ phase, and $K$ stands for the elastic bulk moduli. 
Micro-model for the estimation of $\left\langle\frac{\delta \epsilon_{\gamma}^{\mathrm{eq}}}{\delta \Sigma}\right\rangle_{V_{\gamma}}$. Leblond proposes from a systematic numerical analysis (Leblond et al., 1986b) the following model for the classical plasticity strain rate at constant stress:

$$
\dot{E}_{\Sigma}^{\mathrm{cp}}=\frac{3(1-z)}{2 \sigma_{\gamma}^{y}} \frac{g(z)}{E} S \dot{\Sigma}^{\mathrm{eq}}
$$

Of course the $g$ function is an approximation, but it appears that this term accounts only for a small part of the plastic strain rate and does not need an accurate estimation.

\section{Symmetrical extension of Leblond model in ideal elasto-plastic case}

We now propose a symmetrical extension of the Leblond model taking into account the possibility of plastic flow in the harder phase. The main idea here is simply to consider that not only the $\alpha$ phase could be totally include into the $\gamma$ phase, but that the reverse situation can also occur. When this situation happens, due to the isotropy of the local problem, the $\gamma$ phase could not sustain plastic flow. Hence, in this case, plastic flow is reported to harder phase. We shall discard Hypothesis 2, and even if the $\gamma$ phase elastic yield limit is small with respect to that of $\alpha$ phase we shall take into account a possible plastic flow in the $\alpha$ phase.

\subsection{Treatment of the 'harder' phase associated macro term}

First homogenization step from the Leblond is kept, we only do not remove terms associated to the $\alpha$ phase. the set of equations obtained without mathematical difficulties is presented below:

$$
\left\{\begin{array}{l}
\dot{E}^{\mathrm{tp}}=\frac{3}{2}\left[\frac{1-z}{\sigma_{\gamma}^{y}}\left\langle\frac{\delta \epsilon^{\mathrm{eq}}}{\delta z}\right\rangle_{V_{\gamma}}+\frac{z}{\sigma_{\alpha}^{y}}\left\langle\frac{\delta \epsilon^{\mathrm{eq}}}{\delta z}\right\rangle_{V_{\alpha}}\right] S \dot{z}, \\
\dot{E}_{T}^{\mathrm{cp}}=\frac{3}{2}\left[\frac{1-z}{\sigma_{\gamma}^{y}}\left\langle\frac{\delta \epsilon^{\mathrm{eq}}}{\delta T}\right\rangle_{V_{\gamma}}+\frac{z}{\sigma_{\alpha}^{y}}\left\langle\frac{\delta \epsilon^{\mathrm{eq}}}{\delta T}\right\rangle_{V_{\alpha}}\right] \dot{T}, \\
\dot{E}_{\Sigma}^{\mathrm{cp}}=\frac{3}{2}\left[\frac{1-z}{\sigma_{\gamma}^{y}}\left\langle\frac{\delta \epsilon^{\mathrm{eq}}}{\delta \Sigma}\right\rangle_{V_{\gamma}}+\frac{z}{\sigma_{\alpha}^{y}}\left\langle\frac{\delta \epsilon^{\mathrm{eq}}}{\delta \Sigma}\right\rangle_{V_{\alpha}}\right] \dot{\Sigma} .
\end{array}\right.
$$

We hence obtain a set of homogenized equation able to take into account plasticity in the 'weaker' phase, so that it only remains to determine the relevant micro-model for that part. Since we are keeping the two terms of plastic strain rate in each phase, we have to introduce two micro-model called $\gamma$ and $\alpha$ configuration for calculating respectively plastic flow in $\gamma$ phase and in $\alpha$ phase. In the same manner, we denote by $v_{\lambda}^{\kappa}$ the volume of the $\lambda$ phase in the $\kappa$ configuration, where $\kappa$ is either $\alpha$ or $\gamma$.

\subsection{Inversion of the micro-mechanical model}

\subsubsection{Inversion of the micro-mechanical model for the TRIP term}

The micro-model used for the estimation of the equivalent strain in the $\alpha$ phase is displayed on Fig. 1(left). It's main difference with Leblond one (Leblond et al., 1989b) is that the $\gamma$ phase is now included within the $\alpha$ phase. For this micro-model, even if yield stress of the $\gamma$ phase is lower than that of the $\alpha$ phase, plastic flow can occur only in the $\alpha$ due to the necessary isotropic volume increase of $\gamma$ phase.

Hypothesis 7 and Boundary conditions 2, are used to define the mechanical problem to solve on the configuration, which remain in the same spirit than the preceding case.

Boundary conditions 2. The boundary conditions used to obtain the equivalent strain of the inverted micro-model are:

- behavior at the center is regular. Hence, no plastic flow occurs in the $\gamma$ phase without significant external load;

- continuity of the radial displacement on the boundaries $\gamma-\alpha^{\mathrm{p}}$ and $\alpha^{\mathrm{p}}-\alpha^{\mathrm{el}}$;

- continuity of the radial stress on the boundaries $\gamma-\alpha^{\mathrm{p}}$ and $\alpha^{\mathrm{p}}-\alpha^{\mathrm{el}}$;

- continuity of the circumferential stress on the boundary $\gamma-\alpha^{\mathrm{p}}$;

- null radial stress on the external boundary of the $\alpha$ phase. 
For this micro-model, Leblond et al. (1989b) and Taleb's (Taleb and Sidoroff, 2002) work remain applicable. We can use it to get first a plastification radius, next the equivalent plastic strain rate:

$$
\left\{\begin{array}{ll}
\left\langle\frac{\delta \epsilon^{\mathrm{eq}}}{\delta z}\right\rangle_{v_{\alpha}^{\alpha}}=-2 \frac{\Delta \epsilon_{\gamma \alpha}^{\mathrm{th}}}{z} \ln (1-z) & \text { if } z<z_{\alpha}, \\
\left\langle\frac{\delta \epsilon^{\mathrm{eq}}}{\delta z}\right\rangle_{v_{\alpha}^{\alpha}}=-2 \frac{\Delta \epsilon_{\gamma \alpha}^{\mathrm{th}}}{z} \ln \left(1-z_{\alpha}\right) & \text { if } z \geqslant z_{\alpha}
\end{array} \quad \text { with } z_{\alpha}=\frac{\sigma_{\alpha}^{y}}{\Delta \epsilon_{\gamma \alpha}^{\mathrm{th}}} \frac{4 \mu_{\alpha}+3 K_{\alpha}}{18 K_{\alpha} \mu_{\alpha}} .\right.
$$

Let us underline that the inversion of phase relative position dot not change the growth of plastic zones which always start to develop from the inner towards the outer radius, due to the constant sign of the difference in compactness $\Delta \epsilon_{\gamma \alpha}$.

\subsubsection{Inversion of the micro-mechanical model for the temperature classical plasticity term}

The situation is opposite for this part of the strain rate. We have now to introduce a new hypothesis, because the plasticity now starts from the outer towards the inner radius. We ensure that the two phase remain a continuous medium through assuming that the two phases are perfectly bonded. Under this hypothesis, and working like in the standard case, we obtain:

$$
\left\langle\frac{\delta \epsilon^{\mathrm{eq}}}{\delta T}\right\rangle_{v_{\alpha}^{\alpha}}=-2 \frac{1-z}{z}\left(\alpha_{\gamma}-\alpha_{\alpha}\right) \ln (1-z) .
$$

\subsubsection{Inversion for the mechanic load classical plasticity term}

For $\dot{E}_{\Sigma}^{\mathrm{cp}}$, a different point of view is needed. This term is not only related to isotropic volume variation. It is then not obvious that any plastic flow will occur in the harder phase. Plastic flow may occur in a totally included phase if the mechanical load is not isotropic. Considering this fact it was decided to keep an elastic behavior of the $\alpha$ phase in case of variation of external mechanic load.

\subsection{Consequence for macroscopic terms}

All preceding results can now be combined to obtain the formulation of the macroscopic terms. We consider a representative macroscopic zone that contains the two phases $\alpha$ and $\gamma$. These phases are represented through a set of $N-n$ configuration $\alpha$, and $n$ configurations $\gamma$. The problem is to get a model which respects the global phase proportion independently of the number $n$ and $N$. For that, we will construct configurations that always respect the global proportions, so that, with $V_{\lambda}$ the whole volume of $\lambda$ phase:

$$
V_{\lambda}=n v_{\lambda}^{\gamma}+(N-n) v_{\lambda}^{\alpha}=N v_{\lambda}
$$

Since we consider an infinite number of configurations, the change from the $\gamma$ dominant to a $\alpha$ dominant configuration case can be considered in a continuous way. Introducing $V_{\lambda}^{\bar{\lambda}}$ to denote the whole volume of the $\lambda$ in the $\bar{\lambda}$ configuration, we obtain:

$$
\langle\cdots\rangle_{V_{\lambda}}=\beta\langle\cdots\rangle_{V_{\lambda}^{\gamma}}+(1-\beta)\langle\cdots\rangle_{V_{\lambda}^{\alpha}}, \quad \beta=\frac{n}{N} .
$$

Finally, using the preceding mixture rule, we obtain the macroscopic strain variations:

$$
\left\{\begin{array}{l}
\dot{E}^{\mathrm{tp}}=\beta \dot{E}_{\gamma}^{\mathrm{tp}}+(1-\beta) \dot{E}_{\alpha}^{\mathrm{tp}}, \\
\dot{E}_{T}^{\mathrm{cp}}=3\left(\alpha_{\gamma}-\alpha_{\alpha}\right)\left[\beta \frac{z \ln (z)}{\sigma_{\gamma}^{y}}-(1-\beta) \frac{(1-z) \ln (1-z)}{\sigma_{\alpha}^{y}}\right] S \dot{T}, \\
\dot{E}_{\Sigma}^{\mathrm{cp}}=\frac{3}{2} \frac{\beta}{E} g(z) \frac{1-z}{\sigma_{\gamma}^{y}} S \dot{\Sigma}^{\mathrm{eq}}
\end{array}\right.
$$


with

$$
\begin{cases}\dot{E}_{\gamma}^{\mathrm{tp}}=-3 \frac{\Delta \epsilon_{\gamma \alpha}^{\mathrm{th}}}{\sigma_{\gamma}^{y}} \ln \left(z_{\gamma}\right) & \text { if } z \leqslant z_{\gamma}, \\ \dot{E}_{\gamma}^{\mathrm{tp}}=-3 \frac{\Delta \epsilon_{\gamma \alpha}^{\mathrm{th}}}{\sigma_{\gamma}^{y}} \ln (z) & \text { if } z>z_{\gamma}, \\ \dot{E}_{\alpha}^{\mathrm{tp}}=-3 \frac{\Delta \epsilon_{\gamma \alpha}^{\mathrm{th}}}{\sigma_{\alpha}^{y}} \ln (1-z) & \text { if } z<z_{\alpha}, \\ \dot{E}_{\alpha}^{\mathrm{tp}}=-3 \frac{\Delta \epsilon_{\gamma \alpha}^{\mathrm{th}}}{\sigma_{\alpha}^{y}} \ln \left(1-z_{\alpha}\right) & \text { if } z \geqslant z_{\alpha} .\end{cases}
$$

\subsection{Using the inversion for simulating heating transformation}

Theoretically, the Leblond model was not design to simulate a transformation on heating. Indeed, the main part of the plastic flow is considered to happen at the beginning of the transformation. So, for modeling TRIP phenomenon on heating, it is necessary to consider plastic flow in the $\alpha$ phase, exactly what has been done here. Hence, assuming that interface between $\alpha$ and $\gamma$ phase is perfectly bonded, we obtain evolution equations:

$$
\begin{cases}\dot{E}_{\gamma}^{\mathrm{tp}}=3 \frac{\Delta \epsilon_{\gamma \alpha}^{\mathrm{th}}}{\sigma_{\gamma}^{y}} \ln \left(z_{\gamma}\right) & \text { if } z \leqslant z_{\gamma}, \\ \dot{E}_{\gamma}^{\mathrm{tp}}=3 \frac{\Delta \epsilon_{\gamma \alpha}^{\mathrm{th}}}{\sigma_{\gamma}^{y}} \ln (z) & \text { if } z>z_{\gamma}, \\ \dot{E}_{\alpha}^{\mathrm{tp}}=3 \frac{\Delta \epsilon_{\gamma \alpha}^{\mathrm{th}}}{\sigma_{\alpha}^{y}} \ln (1-z) & \text { if } z<z_{\alpha}, \\ \dot{E}_{\alpha}^{\mathrm{tp}}=3 \frac{\Delta \epsilon_{\gamma \alpha}^{\mathrm{th}}}{\sigma_{\alpha}^{y}} \ln \left(1-z_{\alpha}\right) & \text { if } z \geqslant z_{\alpha} .\end{cases}
$$

\section{4. $\beta(z)$ law for the change of configuration}

The symmetrical extension of the Leblond model introduces a new undetermined function, $\beta(z)$. This law is directly related to the topology of the phase transformation. We could then imagine three main steps, first, at beginning child phase is completely include into parent phase, so $\beta$ is valued to one. Second, at last, the parent phase is completely include into child phase, so $\beta$ vanish. Between these two steps, and behind the lack of experimental observation we take a linear law function of the phase proportion, as illustrated on Fig. 2. So the determination of the $\beta(z)$ law is replaced by the determination of two proportions limits, $z_{0}$ and $z_{1}$. These limits represent phase proportion such that one phase is totally included in the other one. In order to give an estimation of $z_{0}$ and $z_{1}$, we use simplified polyedric geometry. The growth of the other phase is simulated by sphere on the corners. We consider that one phase is included into the other when the radius of these sphere is such that they are in contact, as shown on Fig. 3. This approach is simplified, but give an approximation for $z_{0}$ and $z_{1}$. Results have been gathered in Table 1 .

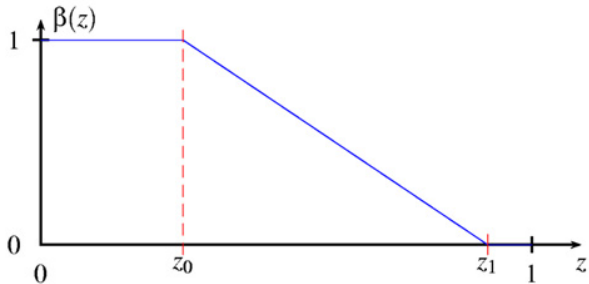

Fig. 2. Linear $\beta$ law for a transformation on cooling

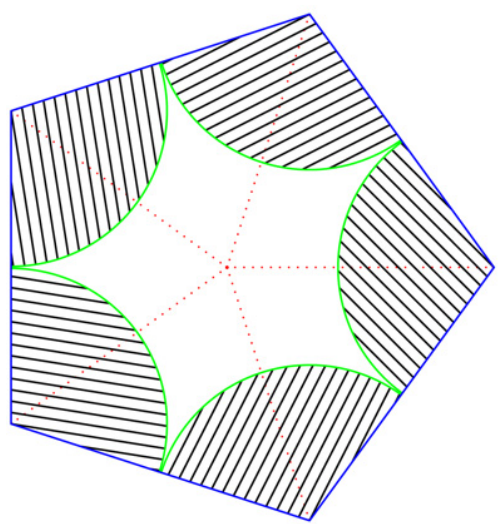

Fig. 3. Simplified polyhedra growing. 
Table 1

Evolution of $z_{0}$ for three-dimensional geometry

\begin{tabular}{llll}
\hline Polyeder & Tetraedra & Hexaedra & Octaedra \\
\hline$z_{0}$ & $74.05 \%$ & $52.36 \%$ & $8.73 \%$ \\
\hline
\end{tabular}

For the simulation, we take $z_{0}=74.05 \%$, like in the tetraedra case, and assuming that there is no reason that behavior acts differently in the other direction, we take $z_{1}=1-z_{0}=25.95 \%$.

\section{Coupling with linear kinematic strain hardening phenomena}

Coupling with kinematic hardening is relevant for such a model since experimentation conducted by Coret et al. (2003) under load reversals shows a typically hardening behavior. The main difficulty here is the estimation of the variation of the hardening parameters. Leblond's work (Leblond et al., 1989a) can be used to predict variation of strain rate.

\subsection{Evolution equations for transformation and classical plastic strain}

The determination of the plastic strain rate remains unchanged. We denote by $A_{\lambda}^{\kappa}$ the center of the macroscopic yield surface of the $\lambda$ phase in the $\kappa$ configuration. Reasoning in the same manner as Leblond et al. (1989a), we obtain constitutive equations:

- TRIP term

$$
\dot{E}^{\mathrm{tp}}=\beta \dot{E}_{\gamma}^{\mathrm{tp}}+(1-\beta) \dot{E}_{\alpha}^{\mathrm{tp}}
$$

with

$$
\begin{cases}\dot{E}_{\gamma}^{\mathrm{tp}}=-3 \frac{\Delta \epsilon_{\gamma \alpha}^{\mathrm{th}}}{\sigma_{\gamma}^{y}} \ln \left(z_{\gamma}\right)\left(S-A_{\gamma}^{\gamma}\right) \dot{z} & \text { if } z \leqslant z_{\gamma}, \\ \dot{E}_{\gamma}^{\mathrm{tp}}=-3 \frac{\Delta \epsilon_{\gamma \alpha}^{\mathrm{th}}}{\sigma_{\gamma}^{y}} \ln (z)\left(S-A_{\gamma}^{\gamma}\right) \dot{z} & \text { if } z>z_{\gamma}, \\ \dot{E}_{\alpha}^{\mathrm{tp}}=-3 \frac{\Delta \epsilon_{\gamma \alpha}^{\mathrm{th}}}{\sigma_{\alpha}^{y}} \ln (1-z)\left(S-A_{\alpha}^{\alpha}\right) \dot{z} & \text { if } z<z_{\alpha}, \\ \dot{E}_{\alpha}^{\mathrm{tp}}=-3 \frac{\Delta \epsilon_{\gamma \alpha}^{\mathrm{th}}}{\sigma_{\alpha}^{y}} \ln \left(1-z_{\alpha}\right)\left(S-A_{\alpha}^{\alpha}\right) \dot{z} & \text { if } z \geqslant z_{\alpha} .\end{cases}
$$

- Classical plasticity terms

$$
\left\{\begin{array}{l}
\dot{E}_{T}^{\mathrm{cp}}=\beta \dot{E}_{T \gamma}^{\mathrm{cp}}+(1-\beta) \dot{E}_{T \alpha}^{\mathrm{cp}} \\
\dot{E}_{\Sigma}^{\mathrm{cp}}=\beta \dot{E}_{\Sigma \gamma}^{\mathrm{cp}}
\end{array}\right.
$$

with

$$
\left\{\begin{array}{l}
\left\{\begin{array}{l}
\dot{E}_{T \gamma}^{\mathrm{cp}}=3 z \frac{\alpha_{\gamma}-\alpha_{\alpha}}{\sigma_{\gamma}^{y}} \ln (z)\left(S-A_{\gamma}^{\gamma}\right) \dot{T} \\
\dot{E}_{T \alpha}^{\mathrm{cp}}=3(z-1) \frac{\alpha_{\gamma}-\alpha_{\alpha}}{\sigma_{\alpha}^{y}} \ln (1-z)\left(S-A_{\alpha}^{\alpha}\right) \dot{T}
\end{array}\right. \\
\dot{E}_{\Sigma \gamma}^{\mathrm{cp}}=\frac{3}{2} \frac{1}{E} g(z) \frac{1-z}{\sigma_{\gamma}^{y}}\left(S-A_{\gamma}^{\gamma}\right) \dot{\Sigma}_{\gamma}^{\mathrm{eq}}
\end{array}\right.
$$

\subsection{Evolution equations for the hardening parameters}

The last step is the estimation of the homogenized hardening parameter evolution for linear kinematic hardening during phase transformation. Each configuration $\lambda$ undergoes a different history. Hence a fictitious front appears 
between the $\alpha$ and $\gamma$ configurations. Expression of $\dot{B}_{\lambda}$, the homogenized hardening parameter in the phase $\lambda$, will be obtained as a function of $\dot{b}_{\lambda}$, the microscopic hardening parameter in the phase $\lambda$, by time differentiation. Hence, taking into account that the front between the two phases is moving:

$$
\frac{\mathrm{d}}{\mathrm{d} t} \int_{V_{\lambda}} b_{\lambda} \mathrm{d} v=\int_{V_{\lambda}} \dot{b}_{\lambda} \mathrm{d} v-\int_{F} b_{\lambda} U_{n} \mathrm{~d} S
$$

where $F$ denote the transformation front and $U_{n}$ the normal velocity.

Hence, we could get for each phase:

$$
\begin{aligned}
\dot{B}_{\gamma}^{\gamma} & =\frac{\mathrm{d}}{\mathrm{d} t}\left[\frac{1}{V_{\gamma}^{\gamma}} \int_{V_{\gamma}^{\gamma}} b_{\gamma} \mathrm{d} v\right] \\
& =\frac{\mathrm{d}}{\mathrm{d} t}\left[\frac{1}{\beta(1-z) V} \int_{V_{\gamma}^{\gamma}} b_{\gamma} \mathrm{d} v\right] \\
& =-\frac{\dot{\beta}(1-z)-\dot{z} \beta}{\beta^{2}(1-z)^{2} V} \int_{V_{\gamma}^{\gamma}} b_{\gamma} \mathrm{d} v+\frac{1}{\beta(1-z) V}\left[\int_{V_{\gamma}^{\gamma}} \dot{b}_{\gamma} \mathrm{d} v-\int_{F_{\gamma}} b_{\gamma} U_{n} \mathrm{~d} S+\int_{F^{*}} b_{\gamma} U_{n}^{*} \mathrm{~d} S\right] .
\end{aligned}
$$

In upper equations, $F_{\lambda}$ represents the transformation front between the two phases in the $\lambda$ configuration. As said before, $F^{*}$ denotes a fictitious front between the $\gamma$ configuration and the $\alpha$ configuration. On the other hand, we have the expressions:

$$
\begin{array}{ccc}
\dot{z}=\frac{\int_{F_{\gamma}} U_{n} \mathrm{~d} S}{V_{\gamma}^{\gamma}+V_{\alpha}^{\alpha}}=(1-z) \frac{\int_{F_{\gamma}} U_{n} \mathrm{~d} S}{V_{\gamma}^{\gamma}}, & \left\langle b_{\gamma}\right\rangle_{F_{\gamma}}=\frac{\int_{F_{\gamma}} b_{\gamma} U_{n} \mathrm{~d} S}{\int_{F_{\gamma}} U_{n} \mathrm{~d} S}, \\
\dot{\beta}=-\frac{\int_{F^{*}} U_{n}^{*} \mathrm{~d} S}{V_{\gamma}^{\gamma}+V_{\gamma}^{\alpha}}=-\beta \frac{\int_{F *} U_{n}^{*} \mathrm{~d} S}{V_{\gamma}^{\gamma}}, & \left\langle b_{\gamma}\right\rangle_{F^{*}}=\frac{\int_{F^{*}} b_{\gamma} U_{n}^{*} \mathrm{~d} S}{\int_{F^{*}} U_{n}^{*} \mathrm{~d} S} .
\end{array}
$$

Since determination of other terms and of microscopic parameters is not of first interest, they are reported in Appendix A. We summarize here main results that are:

$$
\left\{\begin{array}{l}
\dot{B}_{\gamma}^{\gamma}=\frac{1}{1-z}\left[\dot{E}_{T \gamma}^{\mathrm{cp}}+\dot{E}_{\Sigma \gamma}^{\mathrm{cp}}+\dot{E}_{\gamma}^{\mathrm{tp}}\right], \\
\dot{B}_{\alpha}^{\gamma}=-\frac{\dot{z}}{z}\left[B_{\alpha}^{\gamma}-\theta B_{\gamma}^{\gamma}\right], \\
\dot{B}_{\alpha}^{\alpha}=-\frac{\dot{z}}{z}\left[B_{\alpha}^{\alpha}-\theta B_{\gamma}^{\alpha}\right]+\frac{\dot{\beta}}{1-\beta}\left[B_{\alpha}^{\alpha}-B_{\alpha}^{\gamma}\right]+\frac{1}{z}\left[\dot{E}_{T \alpha}^{\mathrm{cp}}+\dot{E}_{\Sigma \alpha}^{\mathrm{cp}}+\dot{E}_{\alpha}^{\mathrm{tp}}\right], \\
\dot{B}_{\gamma}^{\alpha}=\frac{\dot{\beta}}{1-\beta}\left[B_{\gamma}^{\alpha}-B_{\gamma}^{\gamma}\right] .
\end{array}\right.
$$

\subsection{Heating case}

Always keeping on mind that interface between $\alpha$ and $\gamma$ phase is assumed to be perfectly bonded, and working in the same manner than above, we successively get constitutive equations:

$$
\begin{cases}\dot{E}_{\gamma}^{\mathrm{tp}}=3 \frac{\Delta \epsilon_{\gamma \alpha}^{\mathrm{th}}}{\sigma_{\gamma}^{y}} \ln \left(z_{\gamma}\right)\left(S-A_{\gamma}^{\gamma}\right) \dot{z} & \text { if } z \leqslant z_{\gamma}, \\ \dot{E}_{\gamma}^{\mathrm{tp}}=3 \frac{\Delta \epsilon_{\gamma \alpha}^{\mathrm{th}}}{\sigma_{\gamma}^{y}} \ln (z)\left(S-A_{\gamma}^{\gamma}\right) \dot{z} & \text { if } z>z_{\gamma},\end{cases}
$$




$$
\begin{cases}\dot{E}_{\alpha}^{\mathrm{tp}}=3 \frac{\Delta \epsilon_{\gamma \alpha}^{\mathrm{th}}}{\sigma_{\alpha}^{y}} \ln (1-z)\left(S-A_{\alpha}^{\alpha}\right) \dot{z} & \text { if } z<z_{\alpha}, \\ \dot{E}_{\alpha}^{\mathrm{tp}}=3 \frac{\Delta \epsilon_{\gamma \alpha}^{\mathrm{th}}}{\sigma_{\alpha}^{y}} \ln \left(1-z_{\alpha}\right)\left(S-A_{\alpha}^{\alpha}\right) \dot{z} & \text { if } z \geqslant z_{\alpha}\end{cases}
$$

and then evolution equations:

$$
\left\{\begin{array}{l}
\dot{B}_{\gamma}^{\gamma}=\frac{\dot{z}}{1-z}\left[B_{\gamma}^{\gamma}-\theta B_{\alpha}^{\gamma}\right]-\frac{\dot{\beta}}{\beta}\left[B_{\gamma}^{\gamma}-B_{\gamma}^{\alpha}\right]+\frac{1}{1-z}\left[\dot{E}_{\gamma}^{\mathrm{tp}}+\dot{E}_{\Sigma \gamma}^{\mathrm{cp}}+\dot{E}_{T \gamma}^{\mathrm{cp}}\right] \\
\dot{B}_{\alpha}^{\gamma}=-\frac{\dot{\beta}}{\beta}\left[B_{\alpha}^{\gamma}-B_{\alpha}^{\alpha}\right] \\
\dot{B}_{\alpha}^{\alpha}=\frac{1}{z}\left[\dot{E}_{\alpha}^{\mathrm{tp}}+\dot{E}_{\Sigma \alpha}^{\mathrm{cp}}+\dot{E}_{T \alpha}^{\mathrm{cp}}\right] \\
\dot{B}_{\gamma}^{\alpha}=\frac{\dot{z}}{1-z}\left[B_{\gamma}^{\alpha}-\theta B_{\alpha}^{\alpha}\right] .
\end{array}\right.
$$

\section{Experimental comparison}

The law we obtain will now be used to compare simulation and experimental results due to Coret et al. (2003). We shall present one cooling bi-axial, for a bainitic transformation, and one heating uni-axial example. Material and test parameters could be find in Appendix B.

For using the symmetric extension, and also Leblond's model for simulation, we should have access to the evolution of phase proportion during test. In that goal, phase proportion has been obtained from a free dilatometry test. Imposed load coming from test is then shifted so that beginning of the load imposition corresponds to the beginning of the phase change given by free dilatometry.

\subsection{Bi-axial stress and cooling}

This section compares results with a bi-axial stress case. During cooling the test bar is submitted to a constant tensile stress and a variable sinusoidal shear stress. Strain versus temperature is shown on Fig. 4. It can be observed in this figure that, for the longitudinal deformation the new extension give as good results as those obtained with the original Leblond's one but as far as the shear strain is concerned, the new extension give a better agreement with experiments.

The proposed $\beta$ law gives a way to comment obtained results. We would focus on the shearing test, since it's the one which is the more different for the 2 models. The main difference we found is not on the beginning of the transformation, which seems quite reasonable, since the old and the new model response are identical at this time of the transformation.

As the transformation is going on, below $420^{\circ} \mathrm{C}$, the first difference appears on the maximal shear which agree better with experimental data. At that time the behavior of the two phase is take into account, which is certainly responsible for such a behavior.

After, below $350{ }^{\circ} \mathrm{C}$, TRIP is going faster with the new model. This behavior is clearly due to plastification appearing in the harder phase and enable a better agreement with the final deformation.

For the new model, it could be found that characteristic points and tendencies are shifted in temperature. This could be due to some difference of evolution of phase proportion between the stressed test versus the free test giving the phase proportion for the simulation.

\subsection{Heating case}

In this case, the sample is submitted to a constant traction stress during transformation while heating. Comparison between experiment and symmetrical extension model are shown on Fig. 5. It can be seen that even if the value during the test do not agree perfectly with the experiment, the final response, with the ideal elasto-plastic behavior, is near the experimental result. 

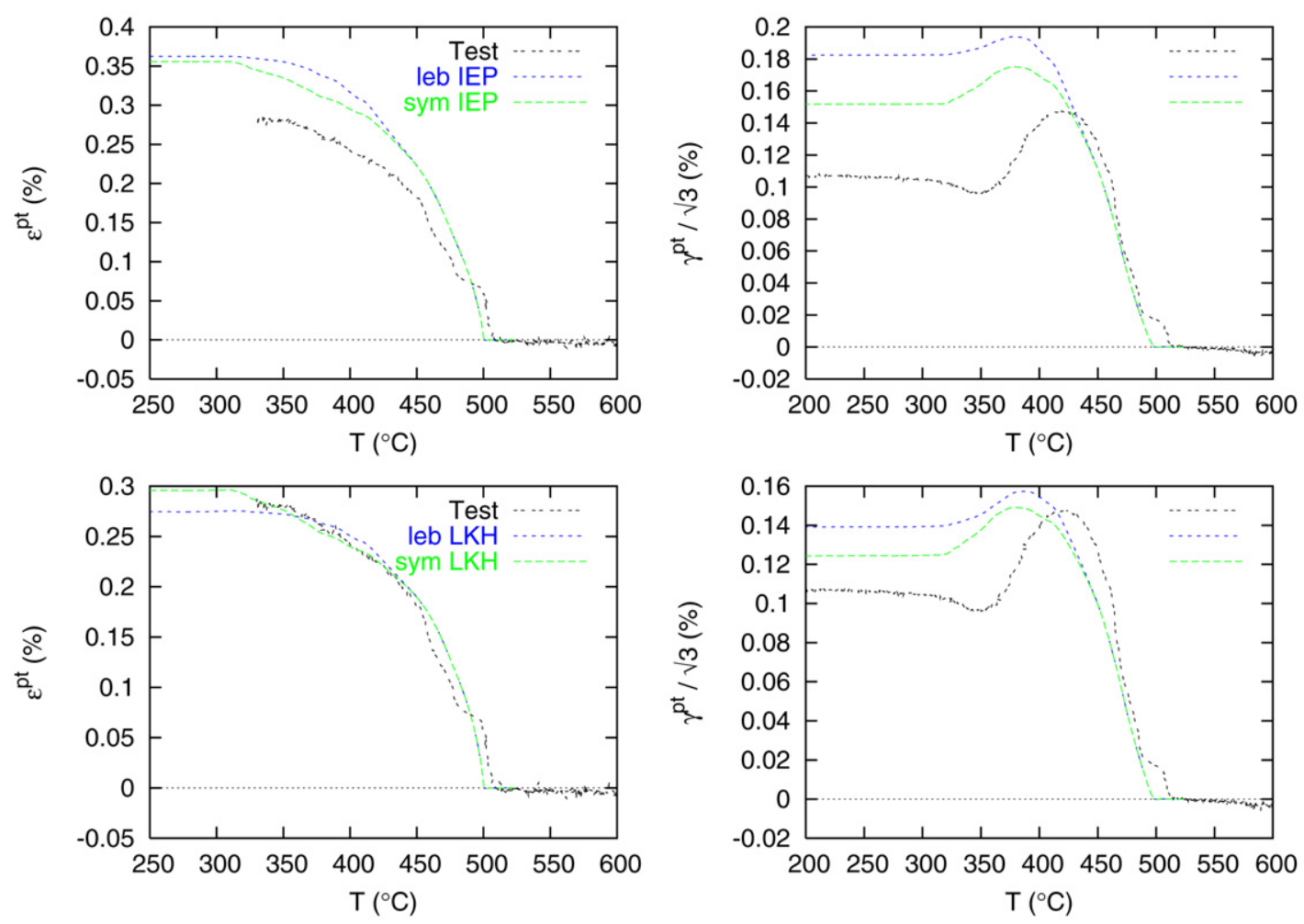

Fig. 4. Bi-axial tension shearing test. leb: Leblond model, sym: symetrical extension, IEP: Ideal Elasto Plastic law, LKH: Linear Kinematic Hardening law.

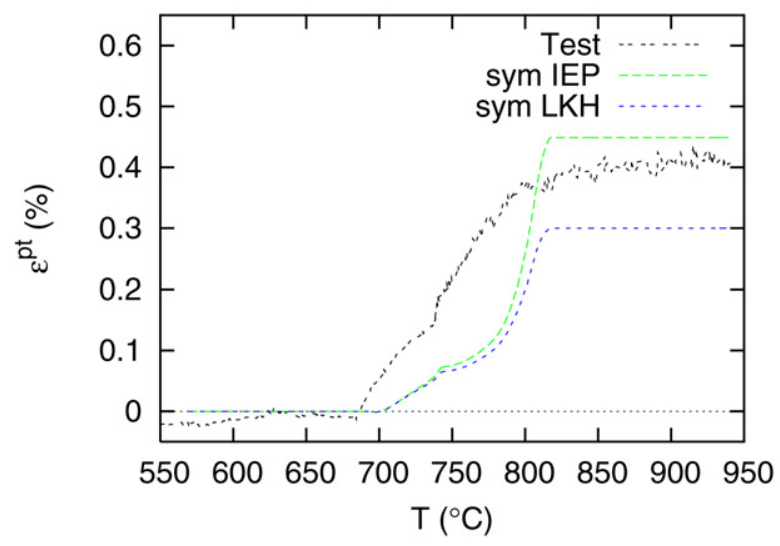

Fig. 5. Uni-axial tension test on heating. sym: symetrical extension, IEP: Ideal Elasto Plastic law, LKH: Linear Kinematic Hardening law.

\section{Conclusion}

As it has been shown in this paper that the Greenwood-Johnson hypothesis about the non-plastification of the weaker phase, in a Leblond model point of view, can be removed. The two first part show how to do it and its implication on macroscopic model. Then, using a mixture rule, all preceding equations can be combined to introduce a model with linear kinematic hardening. This improved model leads to a better understanding of the TRIP phenomenon since it shows that the plastic behavior of the two phases must be take into account

Preceding figures show that the proposed improvement, using the symmetrical extension, do not improve drastically the results. But, for the cases for which this extension is not very efficient, the results remain close to those obtained 
by classical Leblond model. When the results are different from the Leblond's one, the predictions by the symmetrical extension model give definitely more accurate results. Another interesting feature is that the symmetrical extension can be used for heating simulations. This feature is of interest for simulation of multi-pass welding and so on. Coupling with viscosity following Vincent (2002) has been tried but has not led to significant improvements.

\section{Acknowledgement}

Many thanks to Professor R. de Borst for his help to finalize the paper.

\section{Appendix A. Determination of hardening parameters}

Details of the time differentiation for each case are given by:

$$
\begin{aligned}
& \dot{B}_{\gamma}^{\gamma}=\frac{\dot{z}}{1-z}\left[\left\langle b_{\gamma}\right\rangle_{V_{\gamma}^{\gamma}}-\left\langle b_{\gamma}\right\rangle_{F_{\gamma}}\right]-\frac{\dot{\beta}}{\beta}\left[\left\langle b_{\gamma}\right\rangle_{V_{\gamma}^{\gamma}}-\left\langle b_{\gamma}^{\gamma}\right\rangle_{F^{*}}\right]+\left\langle\dot{b}_{\gamma}\right\rangle_{V_{\gamma}^{\gamma}}, \\
& \dot{B}_{\alpha}^{\gamma}=-\frac{\dot{z}}{z}\left[\left\langle b_{\alpha}\right\rangle_{V_{\alpha}^{\gamma}}-\left\langle b_{\alpha}\right\rangle_{F_{\gamma}}\right]-\frac{\dot{\beta}}{\beta}\left[\left\langle b_{\alpha}\right\rangle_{V_{\alpha}^{\gamma}}-\left\langle b_{\alpha}^{\gamma}\right\rangle_{F^{*}}\right]+\left\langle\dot{b}_{\alpha}\right\rangle_{V_{\alpha}^{\gamma}}, \\
& \dot{B}_{\alpha}^{\alpha}=-\frac{\dot{z}}{z}\left[\left\langle b_{\alpha}\right\rangle_{V_{\alpha}^{\alpha}}-\left\langle b_{\alpha}\right\rangle_{F_{\alpha}}\right]+\frac{\dot{\beta}}{1-\beta}\left[\left\langle b_{\alpha}\right\rangle_{V_{\alpha}^{\alpha}}-\left\langle b_{\alpha}^{\alpha}\right\rangle_{F^{*}}\right]+\left\langle\dot{b}_{\alpha}\right\rangle_{V_{\alpha}^{\alpha}}, \\
& \dot{B}_{\gamma}^{\alpha}=\frac{\dot{z}}{1-z}\left[\left\langle b_{\gamma}\right\rangle_{V_{\gamma}^{\alpha}}-\left\langle b_{\gamma}\right\rangle_{F_{\alpha}}\right]+\frac{\dot{\beta}}{1-\beta}\left[\left\langle b_{\gamma}\right\rangle_{V_{\gamma}^{\alpha}}-\left\langle b_{\gamma}^{\alpha}\right\rangle_{F^{*}}\right]+\left\langle\dot{b}_{\gamma}\right\rangle_{V_{\gamma}^{\alpha}} .
\end{aligned}
$$

To conclude, it is now necessary to estimate hardening parameter for each phase and each configuration at a microscopic level.

Hardening parameter for the $\gamma$ configuration. Directly from Leblond's (Leblond et al., 1989b) work, we can write:

- at a point which is not undergoing the transformation, said didn't belong to transformation front $F_{\gamma}$, evolution law is take as usual $\dot{b}_{\lambda}=\dot{\epsilon}_{\lambda}^{\mathrm{p}}$, or, more finely, $\dot{\epsilon}_{\alpha}^{\mathrm{p}}=0$ and $\left\langle\dot{\epsilon}^{\mathrm{p}}\right\rangle_{V_{\gamma}^{\gamma}}=\dot{E}_{T \gamma}^{\mathrm{cp}}+\dot{E}_{\Sigma \gamma}^{\mathrm{cp}}+\dot{E}_{\gamma}^{\mathrm{tp}}$;

- we neglect the influence of the disappearing austenite on the position and velocity of transformation front $\left\langle b_{\gamma}\right\rangle_{F^{\gamma}}=\left\langle b_{\gamma}\right\rangle_{V_{\gamma}^{\gamma}}$;

- memory effect is represented by a memory coefficient $\theta$ which simulate the fact that a part of hardening of disappearing austenite is given to new phase $\left\langle b_{\alpha}\right\rangle_{F^{\gamma}}=\theta\left\langle b_{\gamma}\right\rangle_{F^{\gamma}}=\theta B_{\gamma}^{\gamma}$.

\section{Hardening parameter for the $\alpha$ configuration.}

- at a point which is not undergoing the transformation, said didn't belong to transformation front $F_{\alpha}$, evolution law is take as usual $\dot{b}_{\lambda}=\dot{\epsilon}_{\lambda}^{\mathrm{p}}$, or, more finely, $\dot{\epsilon}_{\gamma}^{\mathrm{p}}=0$ and $\left\langle\dot{\epsilon}^{\mathrm{p}}\right\rangle_{V_{\alpha}^{\alpha}}=\dot{E}_{T \alpha}^{\mathrm{cp}}+\dot{E}_{\Sigma \alpha}^{\mathrm{cp}}+\dot{E}_{\alpha}^{\mathrm{tp}}$;

- we neglect the influence of the disappearing austenite on the position and velocity of transformation front $\left\langle b_{\gamma}\right\rangle_{F^{\alpha}}=\left\langle b_{\gamma}\right\rangle_{V_{\gamma}^{\alpha}}$

- memory effect is represented by a memory coefficient $\theta$ which simulate the fact that a part of hardening of disappearing austenite is given to new phase $\left\langle b_{\alpha}\right\rangle_{F^{\alpha}}=\theta\left\langle b_{\gamma}\right\rangle_{F^{\alpha}}=\theta B_{\gamma}^{\alpha}$.

\section{Hardening parameter when changing of configuration (from $\gamma$ to $\alpha$ ).}

- the $\gamma$ phase in the $\gamma$ configuration is not changed by the inversion of configuration $\left\langle b_{\gamma}^{\gamma}\right\rangle_{F^{*}}=\left\langle b_{\gamma}\right\rangle_{V_{\gamma}^{\gamma}}$;

- the $\alpha$ phase in the $\gamma$ configuration is not changed by the inversion $\left\langle b_{\alpha}^{\gamma}\right\rangle_{F^{*}}=\left\langle b_{\alpha}\right\rangle_{V_{\alpha}^{\gamma}}$;

- the $\gamma$ phase in the $\alpha$ configuration get exactly hardening it had in the precedent configuration $\left\langle b_{\gamma}^{\alpha}\right\rangle_{F^{*}}=\left\langle b_{\gamma}\right\rangle_{V_{\gamma}^{\gamma}}$;

- the $\alpha$ phase in the $\alpha$ configuration get exactly hardening it had in the precedent configuration $\left\langle b_{\alpha}^{\alpha}\right\rangle_{F^{*}}=\left\langle b_{\alpha}\right\rangle_{V_{\alpha}^{\gamma}}$. 
Table B.1

Chemical composition of $16 \mathrm{MnD} 5$ steel

\begin{tabular}{llllllll}
\hline $\mathrm{C}$ & $\mathrm{Si}$ & $\mathrm{Mn}$ & $\mathrm{Ni}$ & $\mathrm{Cr}$ & $\mathrm{Mo}$ & $\mathrm{Cu}$ & $\mathrm{Fe}$ \\
\hline 0.17 & 0.25 & 1.44 & 0.75 & 0.20 & 0.51 & 0.01 & balance \\
\hline
\end{tabular}

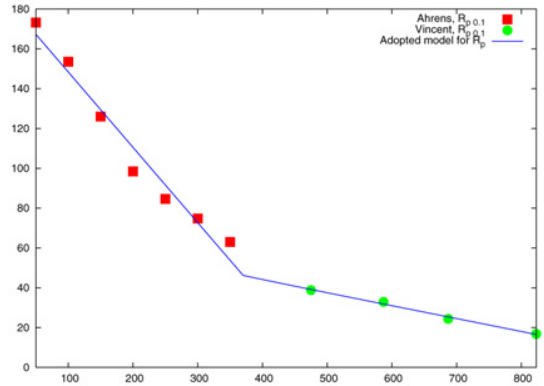

Fig. B.1. $\gamma$ phase yield strength (MPa) versus temperature from Vincent and Ahrens experimental data.

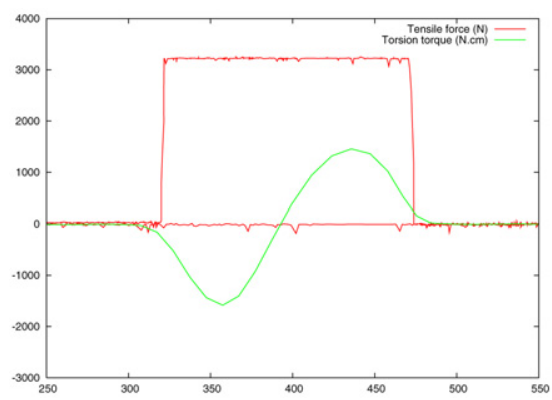

Fig. B.3. Tensile force $(\mathrm{N})$ and torsion torque $(\mathrm{N} \mathrm{cm})$ versus temperature for bi-axial cooling test.

\section{Appendix B. Experimental data}

\section{B.1. Material data}

Tests presented in Section 6 have been conducted on a 16MnD5 steel by Coret. Composition is given in Table B.1. From Ahrens experiments on hyper cooled austenite (Arhens et al., 2004) and Vincent (2002) experiments, yield strength of the tested 16MnD5, could be evaluated as shown on Fig. B.1 for $\gamma$ phase and on Fig. B.2 for $\alpha$ phase.

The hardening parameter could be evaluated to $3700 \mathrm{MPa}$ for $\gamma$ phase and $3000 \mathrm{Mpa}$ for $\alpha$ phase using Grostabussiat-Petit work (Grostabussiat-Petit, 2000).

Thermal dilatation and difference in compactness terms comes from Coret experimentation on 16MND5 steel (Coret et al., 2003). They are valued to:

$$
\alpha_{\gamma}=2310^{-6}, \quad \alpha_{\alpha}=1610^{-6}, \quad \Delta \epsilon_{\gamma \alpha}^{\text {th }}=-1.0610^{-2} .
$$

\section{B.2. Test parameters}

Tensile force and torsion torque applied during cooling and heating tests from (Coret et al., 2003) have been gathered on Figs. B.3 and B.4 respectively. 


\section{References}

Arhens, U., Maier, H.J., Maksoud, A.EL.M., 2004. Stress affected transformation in low alloy steels - factors limiting prediction of plastic strains. J. Phys. IV France 120, 615-623.

Bhadeshia, H., 1995. Mathematical modelling of weld phenomen. In: Cerjak, H. (Ed.), Possible Effects of Stress on Steel Weld Microstructure. pp. 74-77.

Coret, M., Calloch, S., Combescure, A., 2003. Experimental study of the phase transformation plasticity of 16MND5 low carbon steel under multiaxial loading. Int. J. Plasticity.

Fischer, F.D., 1990. A micromechanical model for transformation plasticity in steels. Acta Mater. 38 (8), 1536-1546.

Grostabussiat-Petit, S., 2000. Conséquence mécanique des transformations structurales dans les alliages ferreux. PhD thesis, INSA Lyon.

Greenwood, G.W., Johnson, R.H., 1965. The deformation of metals under small stresses during phase transformations. Proc. R. Soc. $283,403-422$.

Leblond, J.B., Devaux, J., Devaux, J.C., 1989a. Mathematical modelling of transformation plasticity in steel. I. Coupling with strain hardening phenomena. Int. J. Plasticity 5, 573-591.

Leblond, J.B., Devaux, J., Devaux, J.C., 1989b. Mathematical modelling of transformation plasticity in steel. I. Case of ideal-plastic phases. Int. J. Plasticity 5, 551-572.

Leblond, J.B., Mottet, G., Devaux, J.C., 1986a. A theoretical and numerical approach to the plastic behaviour of steels during phase transformations. I. Derivation of general relations. J. Mech. Phys. Solids 34 (4), 395-409.

Leblond, J.B., Mottet, G., Devaux, J.C., 1986b. A theoretical and numerical approach to the plastic behaviour of steels during phase transformations. II. Study of classical plasticity for ideal-plastic phases. J. Mech. Phys. Solids 34 (4), 411-432.

Magee, C.L., 1970. Nucleation of Martensite, Phases Transformations. ASM, Metals Park, OH.

Taleb, L., Sidoroff, F., 2002. Micromechanical modelling of Greenwood-Johnson mechanism in transformation induced plasticity. Int. J. Plasticity.

Vincent, Y., 2002. Simulation numèrique des conséquences metallurgiques et mécaniques induites par une operation de soudage. PhD thesis, INSA Lyon. 